\title{
Resialylation of sialidase-treated sheep and human erythrocytes by Trypanosoma cruzi trans-sialidase: restoration of complement resistance of desialylated sheep erythrocytes
}

\section{Stephen Tomlinson, Lain Pontes de Carvalho, Filip Vandekerckhove and Victor Nussenzweig}

Department of Pathology, New York University Medical Center, 550 First Avenue, New York, NY 10016, USA

Trypanosoma cruzi trans-sialidase (TS) is a recently described enzyme which transfers $\alpha(2-3)$-linked sialic acid from host-derived sialylated glycoconjugates to parasite surface molecules [Schenkman et al. (1991) Cell, 65, 1117]. We report here on the ability of TS to transfer sialic acid from donor sialyl- $\alpha(2-3)$ lactose to sialidase-treated sheep and human erythrocytes. Up to $\sim \mathbf{5 0 \%}$ resialylation of both desialylated red cells could be attained. Resialylation of desialylated sheep erythrocytes restores their resistance to lysis by human complement. This ascribes a possible biological role for $T$.cruzi TS and demonstrates directly that sialic acid is solely responsible for preventing alternative pathway activation of human complement by sheep erythrocytes.

Key words: complement/erythrocytes/sialic acid/sialidase/ trans-sialidase

\section{Introduction}

Trypanosoma cruzi trypomastigotes express on their surface membranes a unique trans-sialidase (TS) that catalyses the transfer of sialic acid from host glycoconjugates to trypomastigote surface molecules (Schenkman et al., 1991). This enzyme differs from previously described sialyl-transferases since it does not utilize CMP-sialic acid as a donor. Known acceptors for T.cruzi TS are saccharides containing terminal $\beta$-linked, but not $\alpha$-linked galactopyranosyl residues (Vandekerckhove et al., 1992).

Although sialic acid appears to be important for cell invasion by T.cruzi, the precise biological role of TS has not yet been defined. Sialic acid is known to be a determinant of complement resistance for some microorganisms (Stevens et al., 1978; Joiner et al., 1984; Mandrell et al., 1990) and it is possible that the sialylation of T.cruzi blood stages (trypomastigotes) with host sialic acid serves such a purpose.

The activation of the alternative pathway of complement on cells by bound $\mathrm{C} 3 \mathrm{~b}$ is influenced by the presence of surfaceassociated sialic acid residues. Sialic acid enhances the affinity of $\mathrm{C} 3 \mathrm{~b}$ for serum factor $\mathrm{H}$, a powerful inhibitor of the assembly of the C3 convertase, i.e. C3bBb (Pangburn, 1989; Meri and Pangburn, 1990). Here we utilize this property of sialic acid to assay for the ability of TS to transfer sialic acid to the plasma membrane of cells, and to increase their resistance to complement lysis.

\section{Results \\ Sialylation of ervthrocytes}

Rabbit, sheep and human erythrocytes were desialylated by treatment with Vibrio cholerae sialidase. Table I shows total membrane sialic acid of the erythrocytes before and after sialidase treatment. As expected, very little sialic acid was detected on rabbit erythrocyte membranes (Pangburn et al., 1980), and the amount found on sheep and human erythrocytes is in accord with published data (Pangburn et al., 1980). Sialidase treatment removed $>90 \%$ of membrane sialic acid from sheep and human erythrocytes. The conditions described for desialylation resulted in maximal release of sialic acid; higher sialidase concentrations or longer incubation times did not result in additional sialic acid release (data not shown).

To determine optimal conditions for the resialylation of erythrocytes, sialidase-treated erythrocytes were incubated with different concentrations of both T.cruzi TS and sialic acid donor, sialyl- $\alpha(2-3)$ lactose. Figure 1 shows that increasing the concentration of TS resulted in increased transfer of sialic acid to the surface of sheep and human erythrocytes. In both cells, only $\sim 50 \%$ of original surface sialylation was attainable (Table I, columns 1 and 3). Increasing the concentration of sialyl- $\alpha(2-3)$ lactose in the trans-sialylation reaction up to $5 \mathrm{mM}$ (at constant enzyme concentration) also resulted in increased sialylation, but again to a maximum of $50 \%$ of initial sialic acid levels (data not shown). The incubation of erythrocytes with either sialyl- $\alpha(2-3)$ lactose or TS alone did not result in any detectable sialylation (not shown). The TS was not able to sialylate rabbit erythrocytes either before or after sialidase treatment.

The amount of sialic acid transferred to erythrocytes under optimal conditions is shown in Table I. The apparent $20 \%$ increase in the level of sialylation of untreated sheep erythrocytes by TS (compare columns 1 and 4 ) is not statistically significant $(P=0.277)$. All erythrocytes used in these experiments were between 2 and 4 weeks old, but storage of erythrocytes for up to 3 months before use did not result in any significant changes in the results obtained.

\section{Sensitivity of erythrocytes to complement}

To demonstrate that resialylation via TS can restore the functional properties of cells with reduced amounts of surface sialic acid, we assayed for their ability to activate the complement cascade. Rabbit erythrocytes, which lack surface sialic acid, are rapidly lysed by the alternative pathway of human complement (Platts-Mills and Ishizaka, 1974) whereas sheep erythrocytes, which bear surface sialic acid, are resistant. Removal of sialic acid from sheep erythrocytes renders them sensitive to human complement (Fearon, 1978; Figure 2).

The effect of resialylation of desialylated sheep erythrocytes by TS was assessed by measuring haemolysis in the presence of human complement. Untreated, desialylated and resialylated sheep erythrocytes were incubated with different concentrations 
Table I. Erythrocyte membrane sialı acid (pmol sıalic acıd/ $10^{6}$ cells) before and after treatment with sialidase and TS

\begin{tabular}{lccrc}
\hline Erythrocyte & Untreated & NA' only & NA then TS & \multicolumn{1}{c}{ TS only } \\
\hline Rabbit & $1.5 \pm 0.13^{\mathrm{c}}$ & $1.3 \pm 0.31$ & $1.3 \pm 0.28$ & $1.6 \pm 0.28$ \\
Sheep & $15.3 \pm 2.3$ & $1.4 \pm 0.31$ & $6.3 \pm 0.57$ & $18.1 \pm 3.1$ \\
Human & $40.8 \pm 3.2$ & $35 \pm 0.61$ & $18.9 \pm 1.14$ & $40.0 \pm 3.5$ \\
\hline
\end{tabular}

"NA = sialidase. Figures represent the maximum amount of desialylation attainable.

${ }^{\text {TTS }}=$ trans-stalidase. Figures represent the maximum amount of resialylation attainable

'Standard deviation $(n=3)$.

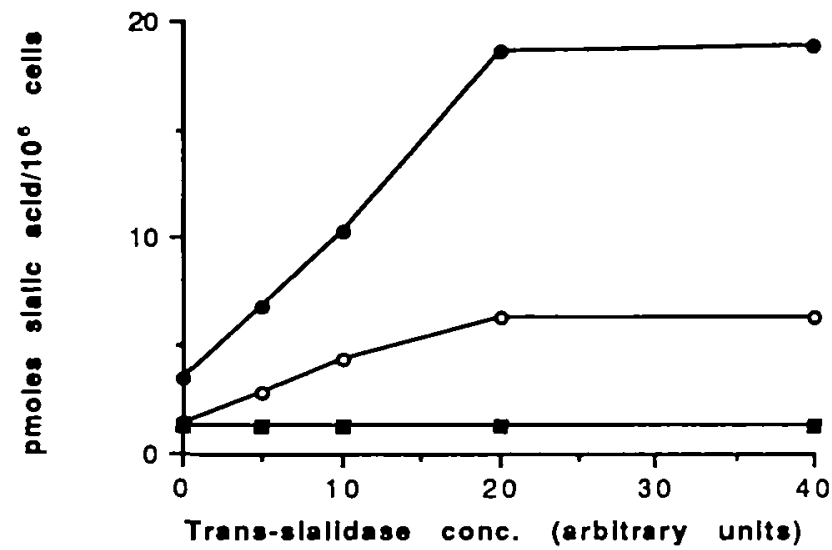

Fig. 1. Resialylation of sialidase-treated erythrocytes by TS. Rabbit ( $\boldsymbol{\nabla})$, sheep $(O)$ and human $(O)$ erythrocytes were initually treated with $V$.cholerae sialıdase. The desialylated erythrocytes were washed and resuspended to $1 \times 10^{9} / \mathrm{ml}$ in HBS containing $2 \mathrm{mM}$ sialyl- $\alpha(2-3)$ lactose. An equal volume of $T . c r u z i$ TS at the indicated concentration was then added and the mixture incubated at $37^{\circ} \mathrm{C}$ for $60 \mathrm{~min}$. Erythrocytes were thoroughly washed and total membrane sialic acid determined

of human serum at $37^{\circ} \mathrm{C}$ for $30 \mathrm{~min}$. Figure 2 shows that partial resialylation of sheep erythrocytes resulted in partial restoration of human complement resistance. Depending on the serum concentration, surface resialylation to $\sim 50 \%$ of original levels resulted in up to a $50 \%$ increase in complement resistance as compared to desialylated erythrocytes. Lower levels of erythrocyte resialylation were less effective in restoring complement resistance, and the level of restoration of complement resistance was approximately proportional to the level of resialylation (Figures 1 and 2). In all cases, haemolysis was due to alternative pathway activation since C2-depleted human serum was as effective as normal human serum, whereas factor B-depleted human serum failed to cause lysis (Figure 2). Nevertheless, TStreated rabbit erythrocytes remained sensitive to human complement (not shown).

\section{Discussion}

Sheep erythrocytes were converted from non-activators to activators, and back to non-activators, of human complement by sialidase and TS treatment, respectively. This provides an additional direct demonstration of the importance of plasma membrane-associated sialic acid in conferring resistance to lysis by complement. That the lysis was mediated by alternative pathway activation was demonstrated by the use of $\mathrm{C} 2-$ or factor B-depleted sera, which are defective in the classical or

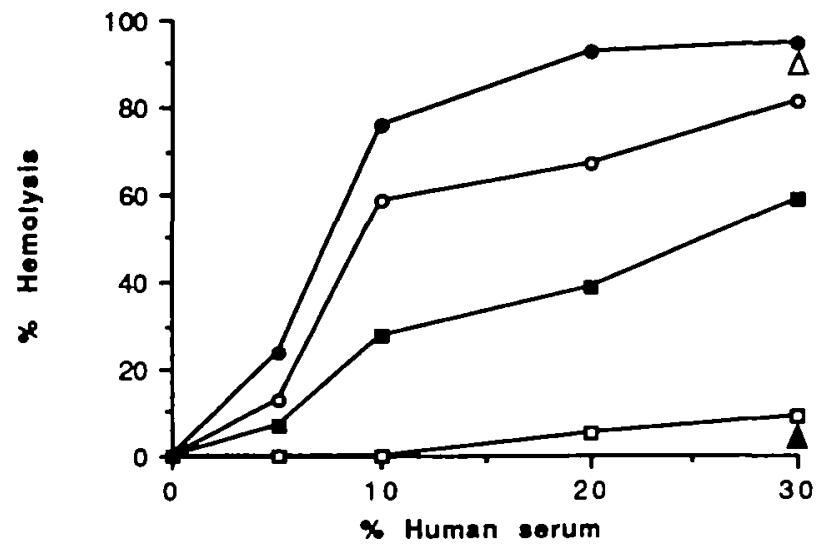

Fig. 2. Haemolysis of untreated desialylated and resialylated sheep erythrocytes. Sheep erythrocytes were desialylated as described, resuspended to $1 \times 10^{\circ} / \mathrm{ml}$ in HBS and then incubated at $37^{\circ} \mathrm{C}$ for $60 \mathrm{~min}$ in an equal volume of HBS (O), or T.cruzi TS diluted $1 / 2(\square)$ or $1 / 8(\mathrm{O})$ Untreated sheep erythrocytes were used as a control ( $\square)$. Single data points are shown for desialylated erythrocytes incubated in $C 2$-depleted $(\Delta)$ and factor B-depleted $(\boldsymbol{\Delta})$ human serum. Following the incubations, erythrocytes

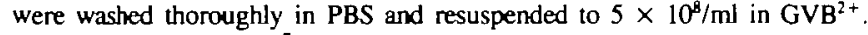
For haemolysis, $5 \times 10^{7}$ erythrocytes were resuspended in the indicated final dilution of human serum, in a total volume of $200 \mu \mathrm{l}$. After $30 \mathrm{~min}$ at $37^{\circ} \mathrm{C}, 1 \mathrm{ml} \mathrm{GVB}{ }^{2+}$ was added and haemolysis determined by measuning the absortance of the supernatants at $412 \mathrm{~nm}$. Complete $(100 \%)$ lysis was achieved by the addition of $\mathrm{H}_{2} \mathrm{O}$ instead of serum to the erythrocytes.

alternative pathways of complement activation, respectively. As shown elsewhere, desialylated human erythrocytes are not lysed by human complement due to homologous restriction conferred by the membrane proteins DAF (Nicholson-Weller et al., 1982) and CD59 (Lachmann, 1991). The majority of human erythrocyte sialic acid is attached to O-linked oligosaccharides of glycophorin A, $50 \%$ being attached by $\alpha(2-3)$ linkage to a terminal $\beta \mathrm{Gal}$ and $50 \%$ being attached by $\alpha(2-6)$ linkage to an internal $\alpha$ GalNAc (Anstee, 1981). Vibrio cholerae sialidase will remove both types of sialic acid. That T.cruzi TS is able to resialylate human erythrocytes only up to $\sim 50 \%$ of their original level is in accord with the fact that the TS is only able to link $\alpha(2-3)$ sialic acid to terminal $\beta$-galactopyranosyl residues (Vandekerckhove et al., 1992). A similar explanation would appear likely for the $50 \%$ resialylation attainable with sheep erythrocytes. Since the level of protection conferred to sheep erythrocytes is approximately proportional to the amount of sialic acid transferred back to desialylated erythrocytes, it appears that the total amount or surface density of sialic acid is the predominant factor controlling alternative pathway activation. Since the type of sialic acid linkage is known to be important for some biological functions (Tomlinson and Taylor, 1985; Foxall et al., 1992; Kelm et al., 1992; Orlandi et al., 1992 ), it is possible, but considered unlikely, that $\alpha(2-3)$-linked and not $\alpha(2-6)$-linked sialic acid is involved in mediating alternative pathway activation. To test this, it would be necessary to correlate complement-mediated haemolysis with $\alpha(2-6)$ resialylation using a $\alpha(2-6)$ sialyl-transferase.

Rabbit erythrocytes were not sialylated by $T$.cruzi TS, most likely due to the absence of suitable acceptors. Indeed, unlike human and sheep erythrocytes, rabbit erythrocytes are known to contain abundant terminal $\alpha \mathrm{Gal}$ (Galili et al., 1987).

This is the first report demonstrating the use of exogenously added purified T.cruzi TS to sialylate heterologous surface membranes. Our findings also provide evidence that the circulating TS found in patients with acute Chagas' disease 
(Affranchino et al., 1989) may alter the structure of cell surfaces and contribute to pathology. The TS may also have some practical applications. Since it can utilize as donors any glycoconjugates bearing sialic acid $\alpha(2-3)$-linked to galactopyranosyl residues, TS appears to be more economical than the CMPsialic acid-dependent $\alpha(2-3)$ sialyl-transferases for this purpose. In addition, the specificity of the T.cruzi enzyme renders it potentially useful in the analysis of sugar composition.

\section{Materials and methods}

\section{Cells and reagents}

Human erythrocytes were isolated from the blood of healthy volunteers drawn into EDTA. Sheep and rabbit erythrocytes were isolated from blood in Alsever solution (Colorado Serum Company, Denver, CO). Erythrocytes were washed four times in phosphate-buffered salıne (PBS) just before use. Protease-free $V$.cholerae sialidase was purchased from Boehringer Mannheım (Indianapolis, IN) and TS was purified from the culture supematant of T.cruzi-infected LLC$\mathrm{MK}_{2}$ cells by affinity chromatography as described previously (Schenkman et al., 1992). Normal human serum was obtained from healthy volunteers and stored at $-80^{\circ} \mathrm{C} \mathrm{C2}$ - and factor B-depleted serum was obtained from Quidel Inc. (San Diego, CA).

\section{Desialylation of erythrocytes}

Erythrocytes were washed in PBS and resuspended to $1 \times 10^{9} / \mathrm{ml}$ in $130 \mathrm{mM}$ $\mathrm{NaCl}, 50 \mathrm{mM} \mathrm{Na}$ acetate (pH 65 ) containing $0.01 \%$ HSA. Equal volumes of erythrocytes and V.cholerae sialidase $(0.5 \mathrm{U} / \mathrm{ml}$ in the above buffer at $\mathrm{pH} 5.5)$ were incubated for $60 \mathrm{~min}$ at $37^{\circ} \mathrm{C}$. Erythrocytes were then washed five times in PBS before resuspension in appropriate buffer for subsequent experiments.

\section{Sialylation of erythrocytes}

Untreated or slalidase-treated erythrocytes were resuspended at $1 \times 10^{9} / \mathrm{ml}$ in $150 \mathrm{mM} \mathrm{NaCl}, 10 \mathrm{mM}$ Hepes ( $\mathrm{pH} 7.0$ ) (HBS) and an equal volume of erythrocytes was mixed with equal volumes of T.cruzi TS at different concentrations. Sialyl- $\alpha(2-3)$ lactose was then added to a final concentration of between 0.1 and $5 \mathrm{mM}$, and the mixture incubated at $37^{\circ} \mathrm{C}$ for $60 \mathrm{~min}$. TS dilutions were made in HBS. Erythrocytes were then washed five times in PBS before resuspension in appropriate buffer for subsequent experiments. Amounts of TS used are indicated in arbitrary units and represent dilutions of a concentrated stock of affinty purified enzyme (see Schenkman et al., 1992).

\section{Sialic acid determination}

Erythrocytes were lysed and the membranes washed five times in $5 \mathrm{mM} \mathrm{Na}$ phosphate buffer ( $\mathrm{pH} 7.8)$. The membrane pellet was resuspended in an equal volume of phosphate buffer, followed by an equal volume of $0.2 \mathrm{M} \mathrm{H}_{2} \mathrm{SO}_{4}$ (0.1 M final concentration). The membranes were then hydrolysed at $80^{\circ} \mathrm{C}$ for $60 \mathrm{~min}$ and the free sialic acid released assayed by an adaptation (Powell and Hart, 1986) of the thiobarbituric acid method (Warren, 1959).

\section{Complement haemolytic assay}

One hundred microlitres of untreated, desialylated or resialylated erythrocytes were resuspended at $5 \times 10^{8} / \mathrm{ml}$ in $\mathrm{GVB}^{2+}$ (gelatin veronal buffered saline containing $0.15 \mathrm{mM} \mathrm{Ca}^{2+}$ and $1 \mathrm{mM} \mathrm{Mg}^{2+}$ ), and mixed with equal volumes of human serum diluted in $\mathrm{GVB}^{2+}$. Incubations were carried out for $30 \mathrm{~min}$ at $37^{\circ} \mathrm{C}$ and the reaction stopped by the addition of $1 \mathrm{ml}$ ice-cold $\mathrm{GVB}^{2+}$. Following centrifugation, the extent of haemolysis was determined by measuring the absorbance of the supernatants at $412 \mathrm{~nm}$. Complete (100\%) lysis was achieved by the addition of $\mathrm{H}_{2} \mathrm{O}$.

\section{Acknowledgements}

We thank C. Huang for expent technical assistance. This work was supported by NIH grant Al32966, by the John D. and Catherine T.MacArthur Foundation, by the United Nations Development Program/World Health Organization Special Program for Research and Training in Tropical Diseases, and by the Rockefeller Foundation. F.V. is a research assistant of the Belgian National Fund for Scientific Research and L.P.C. is a senior investigator of the Oswaldo Cruz Foundation and is a recipient of a grant from the RHAE program, Secretary of Science and Technology, Brazil.

\section{Abbreviations}

$\mathrm{GVB}^{2+}$. gelatin veronal buffered salıne containing $0.15 \mathrm{mM} \mathrm{Ca}^{2+}$ and $1 \mathrm{mM}$ $\mathrm{Mg}^{2+}, \mathrm{PBS}$, płosphate-buffered saline; TS, trans-sialidase.

\section{References}

Affranchino,J.L., Ibanez,C.F, Luquetti,A.O., Rassi,A., Reyes,M.B., Macina,R.A., Aslund,L, Pettersson,U. and Frasch,A.C.C. (1989) Identification of a Trypanosoma cruzi antigen that is shed during the acute phase of Chagas' disease. Mol. Biochem. Parasitol., 34, 221-228.

Anstee,D.J. (1981) The blood group MNSs-active sialoglycoproteins. Semin. Hemanol., 18, 13-31.

Fearon,D T (1978) Regulation of membrane sialic acid of $\beta 1 \mathrm{H}$-dependent decay-dissociation of amplification $\mathrm{C} 3$ convertase of the alternative complement pathway Proc. Natl. Acad. Sci. USA, 75, 1971-1975.

Foxall,C., Watson,S.R., Dowbenko,D., Fennie,C., Lasky,L.A., Kiso,M., Hasegawa,A., Asa,D. and Brandley,B.K. (1992) The three members of the selectin receptor family recognize a common carbohydrate epitope, the sialyl Lewis ${ }^{x}$ oligosaccharide. J. Cell Biol., 117, 895-902.

Galil,,U., Basbaum,C.B., Shohet,S.B., Buehler,J. and Macher,B.A. (1987) Identification of erythrocyte Gal $\alpha 1-3$ Gal glycosphingolipids with a mouse monoclonal antibody, Gal-13. J. Biol. Chem., 262, 4683-4688.

Joiner,K.K., Brown,E.J. and Frank,M.M. (1984) Complement and bacteria. Annu Rev. Microbiol, , 2, 461-491.

Kelm,S., Paulson,J.C., Rose,U., Brossmer,R., Schmid,W., Bandgar,B.P , Schreiner,E., Hartmann, M. and Zbiral,E. (1992) Use of sialic acid analogues to define functional groups involved in binding to the influenza virus hemagglutinin. Eur. J. Biochem, 205, 147-153.

Lachmann,P.J. (1991) The control of homologous lysis. Immunol. Today, 12, 312-315

Mandrell,R.E., Lesse,A.J., Sugai,J.V., Shero,M., Grffiss,J.McL., Cole,J.A., Parsons, N J , Smith,H., Morse,S.A. and Apicella,M A. (1990) In vitro and in vivo modification of Neisseria gonorrhoeae lipooligosaccharide epitope structure by sialylation. J. Exp. Med., 171, 1649-1664.

Men,S. and Pangbum,M.K. (1990) Discrimination between activators and nonactivators of the alternative pathway of complement: regulation via a sialic acid/polyanion binding site on factor $\mathrm{H}$ Proc. Natl. Acad Sal. USA, 87, 3982-3986.

Nicholson-Weller,A., Burge,J., Fearon,D.T., Weller,P.F and Austen,K F. (1982) Isolation of a human erythrocyte membrane glycoprotein with decay accelerating activity for $\mathrm{C} 3$ convertases of the complement system J. Immunol., 129, 184-189.

Orlandi,P.A., Klot,F.W. and Haynes,J.D. (1992) A malaria invasion receptor, the 175-kilodalton erythrocyte binding antigen of Plasmodium falciparum recognizes the terminal NeuSAc( $\alpha 2-3)$ Gal-sequences of glycophorin A. J. Cell Biol., 116, 901-909.

Pangbum,M.K. (1989) Analysis of recognition in the alternatuve pathway of complement. J. Immunol., 142, 2766-2770.

Pangburn,K.M., Morrison,D.C., Schreiber,R.D. and Mueller-Eberhard,H.J. (1980) Activation of the alternative complement pathway: recognition of surface structures on activators by bound C3b. J. Immunol., 124, 977-987.

Platts-Mills,T.A.E. and Ishizaka, K. (1974) Activation of the altemative pathway of human complement by rabbit cells. $J$. Immunol., 113, 348-358.

Powell,L.D and Hart,G.W. (1986) Quantitation of picomole levels of $N$-acetyl and $N$-glycolylneuraminic acids by a HPLC-adaptation of the thiobarbituric acid assay. Anal. Biachem., 157, 179-185.

Schenkman,S., Jiang,M.-S., Hart,G.W. and Nussenzweig,V. (1991) A novel cell surface trans-sialidase of Trypanosoma cruzi generates a stage specific epitope required for invasion of mammalian cells. Cell, 65, 1117-1125.

Schenkman,S., Pontes de Carvalho,L. and Nussenzweig,V. (1992) Trypanosoma cruzi trans-sialidase and sialidase activitues can be mediated by the same enzymes. J. Exp. Med., 175, 567-575.

Stevens,P., Huang,S.N.Y., Welch, W.P. and Young,L.S. (1978) Restricted complement activation by $E$.coli with the $\mathrm{K}-1$ capsular serotype: a possible role in pathogenicity. J. Immunol., 121, 2174-2180.

Tomlinson,S. and Taylor,P.W. (1985) Sialidase associated with coliphage E that specifically depolymerizes the Escherichia coli KI capsular polysaccharide. J. Virol., 55, 374-378

Vandekerckhove,F., Schenkman,S., Pontes de Carvalho,L., Tomlinson,S. Kiso,M., Yoshida,M., Hasegawa,A. and Nussenzweig,V. (1992) Substrate specificity of the Trypanosoma cruzi trans-sialidase. Glycabiology, 2, $541-548$.

Warren,L. (1959) The thiobarbituric acid assay of sialic acids. J. Biol. Chem, 234, 1971-1975.

Received on July 14, 1992; accepted on September 7, 1992 